\title{
Effects of gibberellic acid on water uptake and germination of sweet sorghum seeds under salinity stress
}

\author{
Guanglong Zhu' ${ }^{1,2,3}$, Linlin An², Xiurong Jiao' ${ }^{1}$ Xubing Chen ${ }^{2}$, Guisheng Zhou ${ }^{1,2,3,4^{*}}$, \\ and Neil McLaughlin ${ }^{5}$
}

${ }^{1}$ Yangzhou University, Joint International Research Laboratory of Agriculture and Agri-Product Safety, Yangzhou, 225009, Jiangsu, China.

${ }^{2}$ Yangzhou University, Key Lab of Crop Genetics \& Physiology of Jiangsu Province, Agricultural College, Yangzhou 225001, Jiangsu, China.

${ }^{3}$ Yangzhou University, Jiangsu Co-Innovation Center for Modern Production Technology of Grain Crops, Yangzhou 225009, China.

${ }^{4}$ Yangzhou University, Joint Laboratory in Agricultural Sciences between AAFC, 225009, Jiangsu, China.

"Corresponding author (gszhou@yzu.edu.cn).

${ }^{5}$ Agriculture and Agri-Food Canada, Ottawa Research and Development Center, 960 Carling Ave., Ottawa, Ontario, Canada.

Received: 5 November 2018; Accepted: 11 February 2019; doi:10.4067/S0718-58392019000300415

\begin{abstract}
Sweet sorghum (Sorghum bicolor [L.] Moench) is a potential feedstock crop in biomass energy development, and is much more resistant to saline soils than crops. Healthy seed germination is critical for the growth cycle of plants, and determines the establishment of seedlings and subsequent crop production. High salinity conditions can result in difficulty for seed germination and delays the germination period. So, screening salt-tolerant genotypes and method for healthy seed germination under salinity stress are vital to crop production and food security. Therefore, a controlled study was conducted to explore the interactive amendment effects of exogenous gibberellic acid $\left(\mathrm{GA}_{3}\right)$ and salinity on seed germination process of sweet sorghum. Seeds were presoaked in different levels of $\mathrm{GA}_{3}$ water solutions $(0$, $144,288$, and $576 \mu \mathrm{M})$ and then cultivated in gradient $\mathrm{NaCl}$ solutions $(0,50$ and $100 \mathrm{mM})$. The effects of salinity and external $\mathrm{GA}_{3}$ on seed water uptake and germination characteristics were investigated. Compared with the effects of $0 \mu \mathrm{M} \mathrm{GA}_{3}$ at $0 \mathrm{mM} \mathrm{NaCl}$, slight salt stress of $50 \mathrm{mM} \mathrm{NaCl}$ improved the cumulative water uptake, germination and germination index, but high salinity level of $100 \mathrm{mM} \mathrm{NaCl}$ significantly inhibited these germination traits. However, either $100 \mathrm{mM} \mathrm{NaCl}$ or $576 \mu \mathrm{M} \mathrm{GA}_{3}$ had significantly negative effects on seed cumulative water uptake, cumulative germination, germination index, and length of germ and radicle. The appropriate concentration of $\mathrm{GA}_{3}$ prominently relieved salt stress and improved the seed germination of sorghum seeds, and the optimum concentration for seed germination of sweet sorghum was $288 \mu \mathrm{M} \mathrm{GA}_{3}$ at each salinity level.
\end{abstract}

Key words: $\mathrm{GA}_{3}$, germination, saline stress, sweet sorghum, water uptake.

\section{INTRODUCTION}

Compared to other conventional crops, sweet sorghum (Sorghum bicolor [L.] Moench) can survive in lower quantity of water and fertilizer. It is a potential feedstock crop in biomass energy development. Sweet sorghum is much more resistant to saline soils than corn (Zea mays L.) or sugarcane (Saccharum officinarum L.), which are currently the main bioenergy resources in the world (Almodares et al., 2011). Sweet sorghum crops have a great potential for manufacturing syrups for sweetening food and beverages, carbohydrates, and most importantly, as a raw material for fuel alcohol production worldwide (Ratanavathi et al., 2004). In China, people can take advantage of broad saline lands by planting salt-tolerant 
sweet sorghum varieties. Although it has been found that a number of sweet sorghum varieties can survive under salinity soil condition, however, their seed germination and seedling establishment are still difficult on marginal saline lands.

Currently, more than 900 million hectares or about $20 \%$ of the total agricultural land are affected by salinity around the world. Salinity is becoming an increasingly serious problem limiting crop production worldwide (Munns and Tester, 2008). Salinity can affect crop growth from germination stage, the very beginning of the life cycle of a crop plant. Healthy seed germination plays an important role in the growth cycle of plants, and determines the establishment of seedlings and subsequent crop production (Bahrani and Pourreza, 2012). High salinity conditions can result in difficult seed germination and delays in the germination time (Nyagah and Musyimi, 2009). The inhibition of salinity on seed germination was mainly due to water deficit and/or the toxic influence of ions, such as absorbing excessive $\mathrm{Na}^{+}$and $\mathrm{Cl}$ ions (Murillo et al., 2002).

The water uptake is the first stage preparing for seed germination. Under adequate supply of oxygen and optimal temperature conditions, the most important factor in seed germination is water status. Viable seeds have the ability to break dormancy and begin germination after absorbing enough water. However, water quality varies greatly caused by degrading environments and salinization has an important effect on inhibiting germination and subsequent root elongation (Saberali and Moradi, 2017). It was reported that water uptake of tomato plants declined with increasing salinity, causing significant reductions in morphological and/or physiological parameters, stomatal density and water conductance (Romero et al., 2001).

Plant hormones are active members of plant regulation, and are involved in the induction of plant stress responses (Pedranzani et al., 2003). They make plants adapt to serious abiotic stress conditions, and help crops to improve their tolerance, capacity to adverse environments (Srivastava and Srivastava, 2007). Gibberellic acid $\left(\mathrm{GA}_{3}\right)$ is an important plant hormone, which plays a vital role in regulating the signal pathways, seed germination and plant growth (Cavusoglu and Sulusoglu, 2015). It has been shown that $\mathrm{GA}_{3}$ is related to salinity tolerance of Arabidopsis (Arabidopsis thaliana $\mathrm{L}$.) (Sun, 2008).

There is abundant evidence showed that $\mathrm{GA}_{3}$ has a positive effect on water uptake and germination of crops under normal conditions, but little research is in the literature on sweet sorghum grown in saline soils. Furthermore, the related mechanism of $\mathrm{GA}_{3}$ in regulating germination is still not well documented. Therefore, the objectives of this research were to explore the effects of $\mathrm{GA}_{3}$ amendment on water uptake and germination of sweet sorghum at different salinity levels; and screen the optimal concentrations of $\mathrm{GA}_{3}$ to sweet sorghum germination under saline conditions.

\section{MATERIALS AND METHODS}

A controlled study was conducted in Joint International Research Laboratory of Agriculture and Agri-Product Safety, Ministry of Education of China, Yangzhou University ( $\left.32.30^{\circ} \mathrm{N}, 119.25^{\circ} \mathrm{E}\right)$, Jiangsu Province, China. The seeds of sweet sorghum 'Chuntian 1', kindly provided by Beijing Sangliang Technological Development Center, were used. The variety is relatively salt-tolerant and being popularly grown in China.

\section{Material and cultivation}

The study was arranged in two-factorial randomized complete block design with three replicates. The two factors were $\mathrm{NaCl}\left(0,50\right.$, and $100 \mathrm{mM}$ water solutions) and gibberellic acid $\left(\mathrm{GA}_{3} ; 0,144,288\right.$, and $576 \mu \mathrm{M}$ water solutions). For each replicate of each treatment, 50 uniform and healthy seeds were selected and surface-sterilized using $1 \%$ sodium hypochlorite solution for $10 \mathrm{~min}$, and then rinsed thoroughly six times with deionized water and dried by air, and fresh weight was considered as initial weight. After that, all the seeds were cultivated in Petri dishes with treatment solutions. The diameter of the Petri dish was $9 \mathrm{~cm}$. A double layer of filter paper was placed in each Petri dish, and $7 \mathrm{~mL}$ of different treatment solutions of $\mathrm{NaCl}$ and $\mathrm{GA}_{3}$ were infused. Then the Petri dishes were covered with lids and placed in a germinator (Model ZLC-100, Hangzhou Shuolian Instrument Co., Ltd., Hangzhou, Zhejiang, China) with a natural light 12:12 h diurnal cycle, constant temperature $25^{\circ} \mathrm{C}$ and humidity $60 \%$. In order to maintain the treatment levels, each Petri dish was carefully injected with $5.0 \mathrm{~mL}$ treatment solutions every $8 \mathrm{~h}$ to replenish evaporation and solution absorbed by seeds. The filter paper was changed every $48 \mathrm{~h}$ during the testing period. 


\section{Observations and measurements}

The seeds in each Petri dish were weighed before soaking and during seed water uptake at 8, 16, 24, 32, 40, 48, 56 and $64 \mathrm{~h}$ after the beginning of water imbibition. In order to measure seed cumulative water uptake and water uptake rate, seeds were carefully removed, drained, blotted with absorbent paper, weighed, and, returned to Petri dishes quickly. Germination parameters, including cumulative water uptake, cumulative germination, and germination index at each measurement time were calculated as the following:

Cumulative water uptake $Y_{t}(\%)=\left(X_{t}-X_{0}\right)\left(X_{0}\right)^{-1} \times 100$

where $X_{0}$ is the initial weight of the 50 sweet sorghum seeds $(\mathrm{g})$ and $X_{t}$ is the weight at $\mathrm{t}(\mathrm{h})$.

$$
\text { Cumulative germination }(\%)=S_{t} \times S_{0}^{-1} \times 100
$$

where $S_{t}$ is the number of seeds germinated at $\mathrm{t}(\mathrm{h})$ and $S_{0}$ is the initial number of seeds (50) subjected to the germination test at time $\mathrm{t}=0 \mathrm{~h}$.

$$
\text { Germination index } G_{i}=G_{i-1}+\left(\text { count }_{i}-\text { count }_{i-1}\right) \times S_{0}^{-1} \times\left(t_{i} / 24\right)
$$

where $G_{i}$ is germination index at time $t_{i}(\mathrm{~h})$, count $_{i}$ is the number of seeds germinated at time $\mathrm{t}_{\mathrm{i}}$, count $_{i-1}$ is the number of seeds germinated at time $\mathrm{t}_{\mathrm{i}-1}, S_{0}$ is the number of seeds subjected to the germination test, and $t_{i}$ is observation time.

Seeds were considered to be germinated when radicle length reached approximately $0.2 \mathrm{~mm}$. Both radicle and germ lengths were precisely measured using a vernier caliper after $32 \mathrm{~h}$. The test was terminated after $64 \mathrm{~h}$ in the germinator.

\section{Statistical analysis}

The experiment was designed as a factorial design with two experimental factors (three salinity levels, and four hormones gradients) arranged in a completely randomized design with three replicates. The data of each variable were then subjected to ANOVA with the statistical package of DPS 7.05 for Windows (Tang and Feng, 1997) according to this design. When "F" values were significant, means were separated by the least significant difference test (LSD, P $\leq 0.05)$.

\section{RESULTS}

\section{Cumulative water uptake}

There was nonsignificant effects of salt, $\mathrm{GA}_{3}$ or Salt $\times \mathrm{GA}_{3}$ interaction on cumulative water uptake at the first $32 \mathrm{~h}$ except Salt $\times \mathrm{GA}_{3}$ interaction at $16 \mathrm{~h}$ (Table 1). The effect of salt on cumulative water uptake was nonsignificant at any time point, but the cumulative water uptake was significantly affected by $\mathrm{GA}_{3}$ and Salt $\times \mathrm{GA}_{3}$ at 40 and 48 h, 16 and $48 \mathrm{~h}$, respectively (Table 1). Some of the pairwise comparisons were significantly different from each other as affected by Salt $\times \mathrm{GA}_{3}$ at 16-48 h. Cumulative water uptake was inhibited at whole germination period at high $\mathrm{GA}_{3}$ or high salt. Compared with control, the cumulative water uptake was increased by about $8 \%-12 \%$ at $50 \mathrm{mM}$ salinity level during 8-56 h. However, when salinity was increased to $100 \mathrm{mM} \mathrm{NaCl}$, the cumulative water uptake was decreased by about $14 \%-16 \%$ during the period of 8-48 h. Statistical analysis indicated that the inhibition of seeds cumulative water uptake caused by salinity could be alleviated by application of $\mathrm{GA}_{3}$. With increased concentration of $\mathrm{GA}_{3}$, the cumulative water uptake was gradually increased at low and middle level but decreased at high level of $\mathrm{GA}_{3}$ at $100 \mathrm{mM} \mathrm{NaCl}$. In general, water uptake was promoted by $288 \mu \mathrm{M} \mathrm{GA}_{3}$ but then decreased at $576 \mu \mathrm{M} \mathrm{GA}_{3}$ under each salinity level during whole germination period. Salinity and $\mathrm{GA}_{3}$ treatments showed the most prominent effect during the time period of 32-48 $\mathrm{h}$, the highest cumulative water uptake was recorded at $50 \mathrm{mM} \mathrm{NaCl}$ and $288 \mu \mathrm{M} \mathrm{GA}_{3}$ (Table 2).

\section{Cumulative germination}

The ANOVA showed that cumulative germination of sweet sorghum seeds were significantly influenced by salinity during the germination period, but by $\mathrm{GA}_{3}$ only during $32-48 \mathrm{~h}(\mathrm{P} \leq 0.05$ and $\mathrm{P} \leq 0.01)$. However, Salt $\times \mathrm{GA}_{3}$ had nonsignificant effects on cumulative germination in any growth period (Table 1). Compared with control, $50 \mathrm{mM} \mathrm{NaCl}$ significantly increased the cumulative germination by $23 \%$ at $8 \mathrm{~h}$ and $17 \%$ at $16 \mathrm{~h}$, but then declined with $54 \%$ and $42.2 \%$, respectively, when salinity concentration was increased to $100 \mathrm{mM} \mathrm{NaCl}$. Compared to non-GA $\mathrm{G}_{3}$, cumulative germination was significantly increased at the levels of 144 and $288 \mu \mathrm{M} \mathrm{GA}_{3}$ during 32-48 h, but decreased at $576 \mu \mathrm{M}$ GA 3 at all salinity levels. For example, the cumulative germination declined by $7.5 \%$ from 288 to $576 \mu \mathrm{M} \mathrm{GA}$ at $50 \mathrm{mM} \mathrm{NaCl}$ at $48 \mathrm{~h}$ (Table 3). As a whole, during the germination period of 32-56 h, the highest cumulative germination was presented by applying $288 \mu \mathrm{M} \mathrm{GA}_{3}$ regardless of the salinity levels. 
Table 1. Significance levels of salt, gibberellic acid $\left(\mathbf{G A}_{3}\right)$ and Salt $\times \mathbf{G A}_{3}$ interaction on different germination parameters for sweet sorghum seeds at different measurement times.

\begin{tabular}{|c|c|c|c|c|c|c|c|c|c|}
\hline \multirow[b]{2}{*}{ Parameters } & \multirow[b]{2}{*}{ Factor } & \multicolumn{8}{|c|}{ Time (h) } \\
\hline & & 8 & 16 & 24 & 32 & 40 & 48 & 56 & 64 \\
\hline \multirow[t]{3}{*}{ Cumulative water uptake } & Salt & ns & ns & ns & ns & ns & ns & ns & - \\
\hline & $\mathrm{GA}_{3}$ & ns & ns & ns & ns & $*$ & $*$ & ns & - \\
\hline & Salt $\times \mathrm{GA}_{3}$ & ns & $*$ & ns & ns & ns & $*$ & ns & - \\
\hline \multirow[t]{3}{*}{ Cumulative germination } & Salt & - & $* * *$ & $* *$ & $* *$ & $* *$ & $*$ & $*$ & - \\
\hline & $\mathrm{GA}_{3}$ & - & ns & ns & $* *$ & $*$ & $* *$ & ns & - \\
\hline & Salt $\times \mathrm{GA}_{3}$ & - & ns & ns & ns & ns & ns & ns & - \\
\hline \multirow[t]{3}{*}{ Germination index } & Salt & - & $* * *$ & $* * *$ & $* * *$ & $* * *$ & $* * *$ & $* * *$ & - \\
\hline & $\mathrm{GA}_{3}$ & - & ns & ns & ns & ns & $*$ & ns & - \\
\hline & Salt $\times \mathrm{GA}_{3}$ & - & ns & ns & ns & ns & ns & ns & - \\
\hline \multirow[t]{3}{*}{ Radical length } & Salt & - & - & - & $* * *$ & ns & $* * *$ & $* * *$ & $* * *$ \\
\hline & $\mathrm{GA}_{3}$ & - & - & - & $* * *$ & $* * *$ & $* * *$ & $* * *$ & $* * *$ \\
\hline & Salt $\times \mathrm{GA}_{3}$ & - & - & - & ns & $* * *$ & $* * *$ & $* * *$ & $* * *$ \\
\hline \multirow[t]{3}{*}{ Germ length } & Salt & - & - & - & $* * *$ & $*$ & $* * *$ & $* * *$ & $* *$ \\
\hline & $\mathrm{GA}_{3}$ & - & - & - & ns & $* * *$ & $* * *$ & $* * *$ & $* * *$ \\
\hline & Salt $\times \mathrm{GA}_{3}$ & - & - & - & ns & ns & $* * *$ & $* * *$ & $* * *$ \\
\hline \multirow[t]{3}{*}{ Ratio of radicle and germ lengths } & Salt & - & - & - & * & $* * *$ & $* *$ & $* * *$ & $* * *$ \\
\hline & $\mathrm{GA}_{3}$ & - & - & - & ns & $* *$ & $* * *$ & $* * *$ & $* * *$ \\
\hline & Salt $\times \mathrm{GA}_{3}$ & - & - & - & $\mathrm{ns}$ & $* *$ & $* * *$ & $* * *$ & $* * *$ \\
\hline
\end{tabular}

$*, * *, * * *$ Significant at the $0.05,0.01,0.001$ probability levels, respectively.

ns: Nonsignificant; -: no measurements at that time.

Table 2. Cumulative water uptake of sweet sorghum seeds as influenced by gibberellic acid $\left(\mathrm{GA}_{3}\right)$ amendment at different salinity levels.

\begin{tabular}{crlllllll}
\hline & & \multicolumn{7}{c}{ Cumulative water uptake } \\
\cline { 3 - 9 } Salt & $\mathrm{GA}_{3}$ & \multicolumn{10}{c}{$8 \mathrm{~h}$} & $16 \mathrm{~h}$ & $24 \mathrm{~h}$ & $32 \mathrm{~h}$ & $40 \mathrm{~h}$ & $48 \mathrm{~h}$ & $56 \mathrm{~h}$ \\
\hline $\mathrm{mM}$ & $\mu \mathrm{M}$ & & & & $\%$ & & & \\
\cline { 5 - 9 } 0 & 0 & $32.1 \mathrm{abc}$ & $40.0 \mathrm{ab}$ & $43.6 \mathrm{ab}$ & $47.4 \mathrm{ab}$ & $55.1 \mathrm{bcde}$ & $65.7 \mathrm{bc}$ & $71.6 \mathrm{ab}$ \\
& 144 & $33.2 \mathrm{ab}$ & $39.3 \mathrm{abc}$ & $42.2 \mathrm{ab}$ & $47.9 \mathrm{ab}$ & $57.0 \mathrm{abcd}$ & $68.5 \mathrm{abc}$ & $75.3 \mathrm{ab}$ \\
& 288 & $33.9 \mathrm{a}$ & $40.8 \mathrm{a}$ & $45.9 \mathrm{a}$ & $52.0 \mathrm{a}$ & $62.1 \mathrm{ab}$ & $69.5 \mathrm{abc}$ & $77.7 \mathrm{a}$ \\
& 576 & $31.4 \mathrm{abc}$ & $39.8 \mathrm{ab}$ & $42.9 \mathrm{ab}$ & $46.9 \mathrm{ab}$ & $53.3 \mathrm{de}$ & $62.9 \mathrm{~cd}$ & $73.5 \mathrm{ab}$ \\
50 & 0 & $36.4 \mathrm{a}$ & $43.0 \mathrm{a}$ & $47.3 \mathrm{a}$ & $52.4 \mathrm{a}$ & $61.4 \mathrm{ab}$ & $69.8 \mathrm{abc}$ & $77.7 \mathrm{a}$ \\
& 144 & $34.4 \mathrm{a}$ & $43.4 \mathrm{a}$ & $47.8 \mathrm{a}$ & $51.6 \mathrm{a}$ & $62.5 \mathrm{a}$ & $68.9 \mathrm{abc}$ & $77.7 \mathrm{a}$ \\
& 288 & $34.4 \mathrm{a}$ & $42.4 \mathrm{a}$ & $47.2 \mathrm{a}$ & $52.6 \mathrm{a}$ & $63.6 \mathrm{a}$ & $70.1 \mathrm{abc}$ & $76.0 \mathrm{a}$ \\
& 576 & $26.3 \mathrm{c}$ & $34.0 \mathrm{c}$ & $38.8 \mathrm{~b}$ & $43.8 \mathrm{~b}$ & $54.2 \mathrm{cde}$ & $58.0 \mathrm{~d}$ & $66.6 \mathrm{~b}$ \\
100 & 0 & $27.0 \mathrm{bc}$ & $34.5 \mathrm{bc}$ & $38.8 \mathrm{~b}$ & $42.2 \mathrm{~b}$ & $48.6 \mathrm{e}$ & $56.6 \mathrm{~d}$ & $70.7 \mathrm{~b}$ \\
& 144 & $31.1 \mathrm{abc}$ & $39.6 \mathrm{ab}$ & $43.6 \mathrm{ab}$ & $46.9 \mathrm{ab}$ & $60.5 \mathrm{abc}$ & $70.7 \mathrm{ab}$ & $78.6 \mathrm{a}$ \\
& 288 & $34.1 \mathrm{a}$ & $43.0 \mathrm{a}$ & $46.8 \mathrm{a}$ & $51.2 \mathrm{a}$ & $61.7 \mathrm{ab}$ & $73.2 \mathrm{a}$ & $80.5 \mathrm{a}$ \\
& 576 & $33.2 \mathrm{ab}$ & $42.3 \mathrm{a}$ & $44.7 \mathrm{ab}$ & $51.4 \mathrm{a}$ & $63.0 \mathrm{a}$ & $71.8 \mathrm{ab}$ & $77.0 \mathrm{a}$ \\
\hline
\end{tabular}

Means in the same column and followed by the same letter indicate nonsignificant difference $(\mathrm{P} \geq 0.05)$.

\section{Germination index}

According to the ANOVA, salt stress had a significant effect on germination index, but $\mathrm{GA}_{3}$ had not except at $48 \mathrm{~h}$. The Salt $\times \mathrm{GA}_{3}$ interaction did not significantly affect germination index at any sampling time (Table 1). Compared with control, germination index was slightly decreased at $50 \mathrm{mM} \mathrm{NaCl}$ but it was significantly inhibited at $100 \mathrm{mM} \mathrm{NaCl}$ (Figure 1). At the level of 0 and $100 \mathrm{mM} \mathrm{NaCl}$, germination index was improved by 144 and $288 \mu \mathrm{M} \mathrm{GA}_{3}$, but it was suppressed at $576 \mu \mathrm{M} \mathrm{GA}_{3}$ and $50 \mathrm{mM} \mathrm{NaCl}$. At each salinity level, the highest germination index was recorded at $0 \mathrm{mM}$ $\mathrm{NaCl}$ by $144 \mu \mathrm{M} \mathrm{GA}_{3}, 50 \mathrm{mM} \mathrm{NaCl}$ by $0 \mu \mathrm{M} \mathrm{GA}_{3}$, and $100 \mathrm{mM} \mathrm{NaCl}$ by $288 \mu \mathrm{M} \mathrm{GA}_{3}$ (Figure 1 ). 
Table 3. Cumulative germination of sweet sorghum seeds as influenced by gibberellic acid $\left(\mathrm{GA}_{3}\right)$ amendments at different salinity levels.

\begin{tabular}{|c|c|c|c|c|c|c|c|c|}
\hline \multirow[b]{2}{*}{ Salt } & \multirow[b]{2}{*}{$\mathrm{GA}_{3}$} & \multicolumn{7}{|c|}{ Cumulative germination (\%) } \\
\hline & & $8 \mathrm{~h}$ & $16 \mathrm{~h}$ & $24 \mathrm{~h}$ & $32 \mathrm{~h}$ & $40 \mathrm{~h}$ & $48 \mathrm{~h}$ & $56 \mathrm{~h}$ \\
\hline $\mathrm{mM}$ & $\mu \mathrm{M}$ & & & & $-\%$ & & & \\
\hline \multirow[t]{4}{*}{0} & 0 & $8.7 \mathrm{abc}$ & $39.3 \mathrm{~cd}$ & $70.0 \mathrm{ab}$ & $72.7 \mathrm{bcd}$ & 77.3abcd & 79.3abc & $82.7 \mathrm{abc}$ \\
\hline & 144 & $10.7 \mathrm{ab}$ & $56.7 \mathrm{a}$ & $73.3 \mathrm{a}$ & 74.0abc & $80.0 \mathrm{ab}$ & $80.7 \mathrm{ab}$ & $83.3 \mathrm{ab}$ \\
\hline & 288 & $12.0 \mathrm{a}$ & $53.3 \mathrm{ab}$ & $67.3 \mathrm{abc}$ & $80.0 \mathrm{a}$ & $82.7 \mathrm{a}$ & $84.0 \mathrm{a}$ & $85.3 \mathrm{a}$ \\
\hline & 576 & $10.7 \mathrm{ab}$ & $50.0 \mathrm{abc}$ & $65.3 \mathrm{abcd}$ & $71.3 \mathrm{bcd}$ & 75.3abcde & $76.0 \mathrm{bcd}$ & $80.0 \mathrm{abc}$ \\
\hline \multirow[t]{4}{*}{50} & 0 & $11.3 \mathrm{a}$ & $47.3 \mathrm{a}$ & $68.0 \mathrm{abc}$ & 72.0 & $74.0 \mathrm{bcde}$ & $80.0 \mathrm{ab}$ & $81.3 \mathrm{abc}$ \\
\hline & 144 & $8.0 \mathrm{abc}$ & 45.3abcd & $71.3 \mathrm{ab}$ & $70.7 \mathrm{~cd}$ & 76.7abcd & $80.0 \mathrm{ab}$ & $80.7 \mathrm{abc}$ \\
\hline & 288 & $9.3 \mathrm{abc}$ & $42.0 \mathrm{bcd}$ & $69.3 \mathrm{ab}$ & $77.3 \mathrm{ab}$ & 78.7abc & $81.3 \mathrm{ab}$ & $83.3 \mathrm{ab}$ \\
\hline & 576 & 6.0abcd & $40.7 \mathrm{bcd}$ & $68.7 \mathrm{abc}$ & $68.7 \mathrm{~cd}$ & $73.3 \mathrm{bcde}$ & $74.0 \mathrm{~cd}$ & $79.3 b c$ \\
\hline \multirow[t]{4}{*}{100} & 0 & $4.0 \mathrm{c}$ & 22.7 & $60.7 \mathrm{~cd}$ & 67.3 & 70.0 & 72.7 & $77.3 \mathrm{c}$ \\
\hline & 144 & $4.7 \mathrm{bcd}$ & $37.3 \mathrm{~cd}$ & $57.3 \mathrm{~d}$ & $70.0 \mathrm{~cd}$ & 72.0cde & $76.0 \mathrm{bcd}$ & $78.0 \mathrm{bc}$ \\
\hline & 288 & $3.3 \mathrm{~cd}$ & 32.0de & $67.3 \mathrm{abc}$ & $72.0 \mathrm{bcd}$ & 76.0abcd & $80.7 \mathrm{ab}$ & $80.7 a b c$ \\
\hline & 576 & $1.3 \mathrm{~d}$ & $32.7 \mathrm{de}$ & $63.3 \mathrm{bcd}$ & $68.0 \mathrm{~cd}$ & $68.0 \mathrm{e}$ & $72.7 \mathrm{~d}$ & $77.3 \mathrm{c}$ \\
\hline
\end{tabular}

Means in the same column and followed by the same letter indicate nonsignificant difference $(\mathrm{P} \geq 0.05)$.

Figure 1. Germination index of sweet sorghum seeds calculated at 24 and $48 \mathrm{~h}$ for various combinations of salt and gibberellic acid $\left(\mathrm{GA}_{3}\right)$.

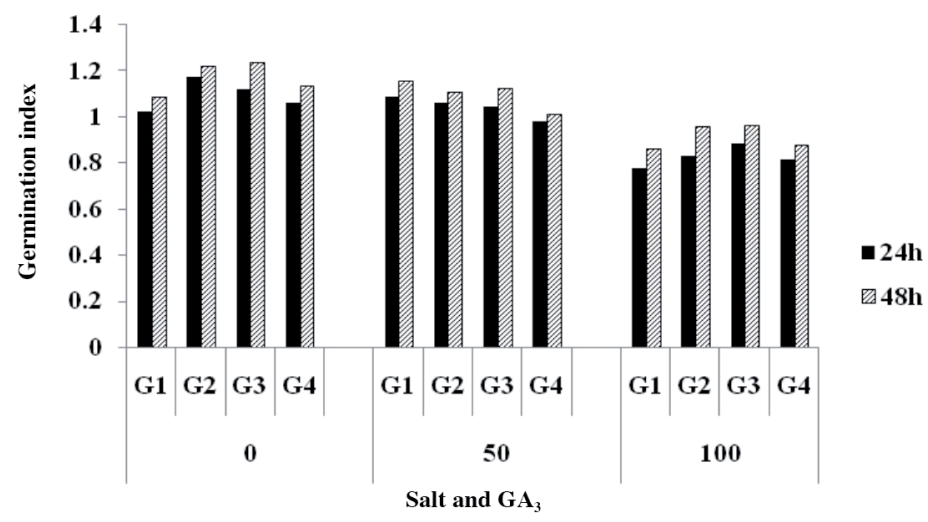

$\mathrm{G} 1, \mathrm{G} 2, \mathrm{G} 3$, and $\mathrm{G} 4$ indicate $\mathrm{GA}_{3}$ concentrations of $0,144,288$ and $576 \mu \mathrm{M}$, respectively. Salinity levels are 0,50 and $100 \mathrm{mM} \mathrm{NaCl}$.

\section{Radicle length}

Salinity, $\mathrm{GA}_{3}$ and Salt $\times \mathrm{GA}_{3}$ significantly affected radicle length mainly after $48 \mathrm{~h}$ water absorption $(\mathrm{P} \leq 0.001)$ (Table 1$)$. Radicle length was improved at the low salinity level but greatly inhibited under high salt stress. Compared with $0 \mathrm{mM}$ $\mathrm{NaCl}$, radicle length was increased by $5 \%-6 \%$ at $50 \mathrm{mM} \mathrm{NaCl}$ during $48-64 \mathrm{~h}$. With rising salinity to $100 \mathrm{mM} \mathrm{NaCl}$, radicle length was significantly decreased by $51 \%-18 \%$ during $8-64 \mathrm{~h}$. The reduction in radicle length caused by salinity was significantly alleviated by external application of 144 and $288 \mu \mathrm{M} \mathrm{GA}_{3}$. Under $100 \mathrm{mM} \mathrm{NaCl}$, radicle length was increased by $26 \%-22 \%$ when applied $288 \mu \mathrm{M} \mathrm{GA}_{3}$ during $40-64 \mathrm{~h}$, but it was prominently inhibited by $576 \mu \mathrm{M} \mathrm{GA}_{3}$ under all salt treatments. Overall, the radical length was prominently enhanced by application of $288 \mu \mathrm{M} \mathrm{GA}_{3}$ during all growth period from 32 to $64 \mathrm{~h}$ under each salinity treatment except at $32 \mathrm{~h}$ with $0 \mathrm{mM} \mathrm{NaCl}$ (Table 4).

\section{Germ length}

The effects of salt, $\mathrm{GA}_{3}$ and Salt $\times \mathrm{GA}_{3}$ were significant on the germ length at 32, 40, and $48 \mathrm{~h}$ after water uptake initiation $(\mathrm{P} \leq 0.001)$ (Table 1). On the average, there was nonsignificant difference in germ length between 0 and $50 \mathrm{mM} \mathrm{NaCl}$ treatments. However, compared to $0 \mathrm{mM} \mathrm{NaCl}$, germ length was decreased by $37 \%-12 \%$ at $100 \mathrm{mM} \mathrm{NaCl}$ during $32-56 \mathrm{~h}$. 
Table 4. Radicle length of sweet sorghum seeds at different times as influenced by gibberellic acid $\left(\mathrm{GA}_{3}\right)$ amendments at different salinity levels.

\begin{tabular}{crllcrl}
\hline & & \multicolumn{5}{c}{ Radicle length } \\
\cline { 3 - 7 } Salt & $\mathrm{GA}_{3}$ & $32 \mathrm{~h}$ & $40 \mathrm{~h}$ & $48 \mathrm{~h}$ & $56 \mathrm{~h}$ & $64 \mathrm{~h}$ \\
\hline $\mathrm{mM}$ & $\mu \mathrm{M}$ & & $\mathrm{mm}$ & & \\
\cline { 3 - 7 } 0 & 0 & $3.7 \mathrm{bc}$ & $5.0 \mathrm{~b}$ & $8.4 \mathrm{bc}$ & $9.4 \mathrm{f}$ & $19.0 \mathrm{~d}$ \\
& 144 & $4.9 \mathrm{a}$ & $4.3 \mathrm{~cd}$ & $7.9 \mathrm{~d}$ & $10.2 \mathrm{e}$ & $19.8 \mathrm{~cd}$ \\
& 288 & $4.4 \mathrm{ab}$ & $5.5 \mathrm{a}$ & $8.8 \mathrm{~b}$ & $13.8 \mathrm{~b}$ & $22.1 \mathrm{~b}$ \\
& 576 & $3.3 \mathrm{~cd}$ & $3.2 \mathrm{e}$ & $5.2 \mathrm{~g}$ & $8.5 \mathrm{~g}$ & $15.8 \mathrm{fg}$ \\
50 & 0 & $2.3 \mathrm{f}$ & $5.1 \mathrm{~b}$ & $8.8 \mathrm{~b}$ & $10.7 \mathrm{~d}$ & $20.2 \mathrm{c}$ \\
& 144 & $3.1 \mathrm{cde}$ & $5.0 \mathrm{~b}$ & $8.1 \mathrm{~cd}$ & $13.8 \mathrm{~b}$ & $21.7 \mathrm{~b}$ \\
& 288 & $3.4 \mathrm{c}$ & $5.7 \mathrm{a}$ & $10.4 \mathrm{a}$ & $15.3 \mathrm{a}$ & $23.9 \mathrm{a}$ \\
& 576 & $2.5 \mathrm{def}$ & $3.2 \mathrm{e}$ & $5.3 \mathrm{fg}$ & $7.5 \mathrm{~h}$ & $16.1 \mathrm{ef}$ \\
& 0 & $1.8 \mathrm{f}$ & $4.2 \mathrm{~cd}$ & $7.1 \mathrm{e}$ & $9.0 \mathrm{f}$ & $15.5 \mathrm{fg}$ \\
& 144 & $2.3 \mathrm{ef}$ & $4.5 \mathrm{c}$ & $8.1 \mathrm{~cd}$ & $11.9 \mathrm{c}$ & $17.1 \mathrm{e}$ \\
& 288 & $2.3 \mathrm{ef}$ & $5.7 \mathrm{a}$ & $8.6 \mathrm{bc}$ & $14.1 \mathrm{~b}$ & $20.0 \mathrm{~cd}$ \\
& 576 & $1.9 \mathrm{f}$ & $4.0 \mathrm{~d}$ & $5.8 \mathrm{f}$ & $8.2 \mathrm{~g}$ & $14.9 \mathrm{~g}$ \\
\hline
\end{tabular}

Means in the same column and followed by the same letter indicate nonsignificant difference $(\mathrm{P} \geq 0.05)$.

The longest germ length was achieved at the concentration of $288 \mu \mathrm{M} \mathrm{GA}_{3}$ under 0 and $50 \mathrm{mM} \mathrm{NaCl}$, but higher GA $\mathrm{G}_{3}$ of $576 \mu \mathrm{M}$ inhibited germ length at each salinity level during all tested periods. However, at the salinity of $100 \mathrm{mM} \mathrm{NaCl}$, the longest germ length was recorded by $144 \mu \mathrm{M} \mathrm{GA}_{3}$ during $40-48 \mathrm{~h}$ and $288 \mu \mathrm{M} \mathrm{GA}_{3}$ during 56-64 $\mathrm{h}$ (Table 5).

\section{The ratio of radicle length to germ length}

The effects of salt, $\mathrm{GA}_{3}$ and Salt $\times \mathrm{GA}_{3}$ on the ratio of radicle length to germ length mainly came up after $40 \mathrm{~h}$ of germination $(\mathrm{P} \leq 0.001)$ (Table 1). The concentration of $144 \mu \mathrm{M} \mathrm{GA}_{3}$ significantly improved radicle length/germ length at $0 \mathrm{mM} \mathrm{NaCl}$ at 32, 48 and $64 \mathrm{~h}$, and at $50 \mathrm{mM} \mathrm{NaCl}$ during all germination period except at $48 \mathrm{~h}$. However, under $100 \mathrm{mM} \mathrm{NaCl}$, radicle length/germ length was promoted by $288 \mu \mathrm{M} \mathrm{GA}_{3}$ at 32 and $40 \mathrm{~h}$, and by $576 \mu \mathrm{M} \mathrm{GA}_{3}$ at 56 and $64 \mathrm{~h}$ (Table 6).

Table 5. Germ length of sweet sorghum seeds at different times as influenced by gibberellic acid $\left(G A_{3}\right)$ amendments at different salinity levels.

\begin{tabular}{crlllll}
\hline & & \multicolumn{5}{c}{ Germ length } \\
\cline { 3 - 7 } Salt & $\mathrm{GA}_{3}$ & & \multicolumn{5}{c}{$32 \mathrm{~h}$} & $40 \mathrm{~h}$ & $48 \mathrm{~h}$ & $56 \mathrm{~h}$ & $64 \mathrm{~h}$ \\
\hline $\mathrm{mM}$ & $\mu \mathrm{M}$ & & & $\mathrm{mm}$ & & \\
0 & 0 & $1.9 \mathrm{abcd}$ & $2.7 \mathrm{bc}$ & $4.2 \mathrm{~cd}$ & $5.7 \mathrm{e}$ & $13.5 \mathrm{~cd}$ \\
& 144 & $2.1 \mathrm{ab}$ & $2.8 \mathrm{ab}$ & $3.8 \mathrm{~d}$ & $7.1 \mathrm{~d}$ & $11.8 \mathrm{ef}$ \\
& 288 & $2.5 \mathrm{a}$ & $3.2 \mathrm{a}$ & $5.2 \mathrm{a}$ & $11.0 \mathrm{a}$ & $16.4 \mathrm{a}$ \\
& 576 & $1.7 \mathrm{bcde}$ & $2.2 \mathrm{de}$ & $3.2 \mathrm{e}$ & $5.6 \mathrm{e}$ & $11.9 \mathrm{ef}$ \\
50 & 0 & $1.4 \mathrm{cde}$ & $2.5 \mathrm{bcd}$ & $4.1 \mathrm{~cd}$ & $6.0 \mathrm{e}$ & $13.8 \mathrm{c}$ \\
& 144 & $1.9 \mathrm{abc}$ & $2.3 \mathrm{cde}$ & $4.6 \mathrm{~b}$ & $6.0 \mathrm{e}$ & $11.4 \mathrm{f}$ \\
& 288 & $1.7 \mathrm{bcde}$ & $2.9 \mathrm{ab}$ & $5.4 \mathrm{a}$ & $8.5 \mathrm{c}$ & $14.2 \mathrm{bc}$ \\
& 576 & $1.4 \mathrm{cde}$ & $1.9 \mathrm{e}$ & $3.1 \mathrm{e}$ & $4.4 \mathrm{fg}$ & $12.4 \mathrm{e}$ \\
& 0 & $1.2 \mathrm{e}$ & $2.1 \mathrm{de}$ & $3.2 \mathrm{e}$ & $5.0 \mathrm{f}$ & $12.7 \mathrm{de}$ \\
& 0 & $1.3 \mathrm{de}$ & $2.9 \mathrm{ab}$ & $4.2 \mathrm{bc}$ & $6.2 \mathrm{e}$ & $12.7 \mathrm{de}$ \\
& 144 & $1.3 \mathrm{de}$ & $2.8 \mathrm{ab}$ & $4.0 \mathrm{~cd}$ & $9.4 \mathrm{~b}$ & $15.1 \mathrm{~b}$ \\
& 288 & $1.4 \mathrm{cde}$ & $2.0 \mathrm{e}$ & $3.3 \mathrm{e}$ & $4.3 \mathrm{~g}$ & $9.0 \mathrm{~g}$ \\
\hline
\end{tabular}

Means in the same column and followed by the same letter indicate nonsignificant difference $(\mathrm{P} \geq 0.05)$. 
Table 6. The ratio of radicle and germ length of sweet sorghum seeds as influenced by gibberellic acid (GA $\left.A_{3}\right)$ amendment at different salinity levels.

\begin{tabular}{|c|c|c|c|c|c|c|}
\hline \multirow[b]{2}{*}{ Salt } & \multirow[b]{2}{*}{$\mathrm{GA}_{3}$} & \multicolumn{5}{|c|}{ Radicle length/Germ length } \\
\hline & & $32 \mathrm{~h}$ & $40 \mathrm{~h}$ & $48 \mathrm{~h}$ & $56 \mathrm{~h}$ & $64 \mathrm{~h}$ \\
\hline $\mathrm{mM}$ & $\mu \mathrm{M}$ & & & & & \\
\hline \multirow[t]{4}{*}{0} & 0 & $2.12 \mathrm{ab}$ & $1.92 \mathrm{abc}$ & $2.03 \mathrm{bc}$ & $1.67 \mathrm{~cd}$ & $1.41 \mathrm{~cd}$ \\
\hline & 144 & $2.61 \mathrm{a}$ & $1.57 \mathrm{~d}$ & $2.11 \mathrm{ab}$ & $1.46 \mathrm{e}$ & $1.67 \mathrm{~b}$ \\
\hline & 288 & $1.82 \mathrm{bc}$ & $1.74 \mathrm{bcd}$ & $1.69 \mathrm{e}$ & $1.26 \mathrm{f}$ & $1.35 \mathrm{de}$ \\
\hline & 576 & $2.04 \mathrm{abc}$ & $1.51 \mathrm{~d}$ & $1.63 \mathrm{e}$ & $1.52 \mathrm{de}$ & $1.33 \mathrm{de}$ \\
\hline \multirow[t]{4}{*}{50} & 0 & $1.73 \mathrm{bc}$ & $2.10 \mathrm{a}$ & $2.18 \mathrm{ab}$ & $1.81 \mathrm{bc}$ & $1.48 \mathrm{c}$ \\
\hline & 144 & $2.09 \mathrm{ab}$ & $2.21 \mathrm{a}$ & $1.77 \mathrm{de}$ & $2.30 \mathrm{a}$ & $1.91 \mathrm{a}$ \\
\hline & 288 & $2.09 \mathrm{ab}$ & $2.01 \mathrm{ab}$ & $1.96 \mathrm{c}$ & $1.81 \mathrm{bc}$ & $1.69 \mathrm{~b}$ \\
\hline & 576 & $1.83 \mathrm{bc}$ & $1.68 \mathrm{ad}$ & $1.75 \mathrm{e}$ & $1.71 \mathrm{c}$ & $1.31 \mathrm{de}$ \\
\hline \multirow[t]{4}{*}{100} & 0 & $1.77 \mathrm{bc}$ & $2.01 \mathrm{ab}$ & $2.25 \mathrm{a}$ & $1.80 \mathrm{bc}$ & $1.23 \mathrm{e}$ \\
\hline & 144 & $1.95 \mathrm{bc}$ & $1.58 \mathrm{~d}$ & $1.92 \mathrm{~cd}$ & $1.92 \mathrm{~b}$ & $1.35 \mathrm{de}$ \\
\hline & 288 & $2.12 \mathrm{ab}$ & $2.08 \mathrm{a}$ & $2.15 \mathrm{ab}$ & $1.49 \mathrm{e}$ & $1.32 \mathrm{de}$ \\
\hline & 576 & $1.47 \mathrm{c}$ & $2.04 \mathrm{a}$ & $1.77 \mathrm{de}$ & 1.94 & $1.67 \mathrm{~b}$ \\
\hline
\end{tabular}

Means in the same column and followed by the same letter indicate nonsignificant difference $(\mathrm{P} \geq 0.05)$.

\section{DISCUSSION}

Salinity inhibits water uptake, delays seed germination, slows down growth rate, changes metabolic and reduces biomass production (Munns, 2002). In most cases, salinity does harm plant survival by disturbing different plant mechanisms (Tavakkoli et al., 2010). Salinity at higher levels usually causes both hypertonic and hyperosmotic stresses and can lead to plant death. These effects may cause membrane damage, nutrient imbalance, altered levels of growth regulators, enzymatic inhibition and metabolic dysfunction (Sudhir and Murthy, 2004), changes in C and N metabolism (Kim et al., 2004), and decreased photosynthesis rate, which ultimately leads to plant death (Mahajan and Tuteja, 2005; Hasanuzzaman et al., 2012). Conventional crops, such as wheat and rice, are sensitive to high salinity conditions and their biomass production and yield gain are impeded sharply at high salinity levels (Bahrani and Haghjoo, 2011).

However, as for sweet sorghum, it has been proved to be more tolerant to salt stress as compared with conventional crops aforementioned (Ratanavathi et al., 2004), but the poor germination under severe salt stress is still a crucial problem to limit sweet sorghum production. An increasing evidence showed that the growth of sorghum is seriously restrained at 250 $\mathrm{mM}$ rather than at $125 \mathrm{mM} \mathrm{NaCl}$ (Ibrahim, 2004). In the present study, cumulative water uptake, cumulative germination and germination index were all exhibited with similar tendencies at $100 \mathrm{mM} \mathrm{NaCl}$. These results are consistent with Yang and $\mathrm{Li}$ (2014). These damage to seed germination and seedling growth is closely related to $\mathrm{Na}^{+}$accumulation. Excessive accumulation of $\mathrm{Na}^{+}$can cause a range of osmotic and metabolic problems for plants (Hoai et al., 2003). Most toxic effects of $\mathrm{NaCl}$ can be attributed to $\mathrm{Na}^{+}$toxicity that can result in the dormancy of seeds and delay germination. It is known that toxic effects of $\mathrm{Na}^{+}$are largely due to its ability to compete with $\mathrm{K}^{+}$for binding sites, essential for cellular function (Yildirim et al., 2009). As a major plant macronutrient, K plays important roles on stomatal behavior, osmoregulation, enzyme activity, cell expansion, neutralization of non-diffusible negatively charged ions, and membrane polarization (Qin et al., 2010). On the other hand, $\mathrm{Na}$ and $\mathrm{Cl}$ ions can enter into the cells and have direct toxic effects on cell membranes, as well as on metabolic activities in the cytosol (Cha-Um and Kirdmanee, 2010).

On the contrary, slight salinity improved germination parameters at the level of $50 \mathrm{mM} \mathrm{NaCl}$. Similar results were found by other scientists (Nimir et al., 2017). There is evidence that low salinity sometimes stimulates photosynthesis of Bruguiera parviflora. Parida et al. (2004) observed that the rate of photosynthesis increased at low salinity while decreased at high salinity. But the related mechanism is still not clear.

The capacity of crops tolerant to stress can be improved by a number of ways, including selection and breeding, genetic modifications, and use of osmoprotectants and growth regulating substances (Parida et al., 2004). In this regard, attention has come to be focused on the use of plant growth regulators, such as $\mathrm{GA}_{3}$, kinetin, and salicylic acid, which are known 
to regulate plant responses to adverse external environments and to regulate the expression of a number of stress-induced genes. At high salinity levels, the germination of sweet sorghum is deteriorated. The osmotic regulation, together with the toxic effects of $\mathrm{Na}^{+}$and $\mathrm{Cl}$ ions, reduces water uptake and causes an imbalance of essential nutrients during seed germination (Willenborg et al., 2004). In the present study, we observed that the cumulative water uptake, cumulative germination, germination index and the length of radicle and germ of sweet sorghum seeds were significant improved by applying 144 and $288 \mu \mathrm{M} \mathrm{GA}_{3}$. Similar results were found in cotton (Gossypium barbadense L.) and castor (Ricinus communis L.) seeds when $\mathrm{GA}_{3}$ was amended at appropriate concentrations (Zhou et al., 2014). Also with wheat seeds, the greatest improvement in seed germination was achieved when seeds were presoaked in $50 \mathrm{mg} \mathrm{GA}_{3} \mathrm{~L}^{-1}(\mathrm{Parashar}$ and Varma, 1988). As for sweet sorghum, the optimum $\mathrm{GA}_{3}$ concentration to promote seed germination is $288 \mu \mathrm{M} \mathrm{GA}_{3}$ in this study. At this level, most of the parameters were improved under each salinity levels during different germination periods (Tables 2-6).

The probable mechanism is that $\mathrm{GA}_{3}$ can break seed dormancy, stimulate seed embryos, thereby promote plant metabolic reactions, repair the integrity of damaged cell and improve seed viability. Nimir et al. (2017) reported that $\mathrm{GA}_{3}$ caused a reduction in $\mathrm{Na}^{+}$content and partly decreased the content of other ions. These results agreed with those of Kaya et al. (2010), who reported that stressed maize plants significantly accumulated less $\mathrm{Na}^{+}$upon application of $\mathrm{GA}_{3}$. In another study, application of $\mathrm{GA}_{3}$ counteracted the adverse effects of $\mathrm{NaCl}$ salinity on relative water content, electrolyte leakage, and chlorophyll content (Ahmad et al., 2011).

According to previous studies, external seed treatment with $\mathrm{GA}_{3}$ could be a possible method of reversing the effects of salt stress (Tuna et al., 2008). However, when $\mathrm{GA}_{3}$ level was increased to $576 \mu \mathrm{M}$, most germination parameters were suppressed as shown in the present study (Tables 2-6). This results indicated that application of low levels of $\mathrm{GA}_{3}$ can regulate plant growth and have positive effects, but high levels of $\mathrm{GA}_{3}$ may have opposite effects. This result is similar to Baskin et al. (1998), who reported that high level of $\mathrm{GA}_{3}$ reduced the ratio of radicle length to germ length, but the suitable $\mathrm{GA}_{3}$ concentration improved the growth rate of the germ relative to the radicle. The application of $288 \mu \mathrm{M} \mathrm{GA}_{3}$ can relieve the harmful effects of salinity on water uptake and germination of sweet sorghum. Nevertheless, these results need to be further confirmed in field environment due to the difference in soil environment and $\mathrm{NaCl}$ water solution.

\section{CONCLUSION}

Sweet sorghum can be tolerant to mild salinity stress, it can be recognized as one of the excellent candidate crops used to exploit the area of coastal shoaly land. Low concentrations of salinity and gibberellic acid $\left(\mathrm{GA}_{3}\right)$ can enhance water absorption and germination of sweet sorghum, but high levels of these treatments can bring the opposite effects. The $288 \mu \mathrm{M} \mathrm{GA}_{3}$ is the optimum concentration which can be applied to promote seed germination of sweet sorghum under salinity stress conditions.

\section{ACKNOWLEDGEMENTS}

This study was financially supported in part by Jiangsu Provincial Key R\&D Program (BE2016345), China National Key R\&D Program (SQ2017YFNC050027), Jiangsu Provincial Independent Agriculture Innovation Program (CX(16)1005-5), the Natural Science Foundation of Jiangsu Province of China (BK20180923), the Natural Science Foundation of Jiangsu Higher Education Institutions of China (17KJB210008), and Open Research Project of Joint International Research Laboratory of Agriculture and Agri-Product Safety, the Ministry of Education of China; Yangzhou University (JRK2018003).

Guanglong Zhu and Linlin An contributed equally to this paper and are considered first coauthors.

\section{REFERENCES}

Ahmad, P., Nabi, G., and Ashraf, M. 2011. Cadmium-induced oxidative damage in mustard plants [Brassica juncea (L.) Czern. \& Coss.] can be alleviated by salicylic acid. South African Journal of Botany 77:36-44.

Almodares, A., Hadi, M.R., and Kharazian, Z.A. 2011. Sweet sorghum: salt tolerance and high biomass sugar crop. p. $441-460$. In Matovic, M. (ed.) Biomass-detection, production and usage. InTech, Rijeka, Croatia. doi:10.5772/19044. 
Bahrani, A., and Haghjoo, M. 2011. Response of some wheat (Triticum aestivum L.) genotypes to salinity at germination and early seedling growth stages. World Applied Sciences Journal 13:887-897.

Bahrani, A., and Pourreza, J. 2012. Gibberellic acid and salicylic acid effects on seed germination and seedlings growth of wheat (Triticum aestivum L.) under salt stress condition. World Applied Sciences Journal 18:633-641.

Baskin, J.M., Nan, X.Y., and Baskin, C.C. 1998. A comparative study of seed dormancy and germination in an annual and a perennial species of Senna (Fabaceae). Seed Science Research 8:501-512.

Cha-Um, S., and Kirdmanee, C. 2010. Effect of glycine betaine on proline, water use, and photosynthetic efficiencies, and growth of rice seedlings under salt stress. Turkish Journal of Agriculture and Forestry 34:517-527.

Cavusoglu, A., and Sulusoglu, M. 2015. Effects of gibberellic acid $\left(\mathrm{GA}_{3}\right)$, indole-3-acetic acid (IAA) and water treatments on seed germination of Melia azedarach L. Scientific Papers. Series B, Horticulture 59:319-326.

Hasanuzzaman, M., Hossain, M.A., da Silva, J.A.T., and Fujita, M. 2012. Plant response and tolerance to abiotic oxidative stress: antioxidant defense is a key factor//Crop stress and its management: Perspectives and strategies. p. 261-315. Springer, Dordrecht, The Netherlands.

Hoai, N.T.T., Shim, I.S., Kobayashi, K., and Kenji, U. 2003. Accumulation of some nitrogen compounds in response to salt stress and their relationships with salt tolerance in rice (Oryza sativa L.) seedlings. Plant Growth Regulation 41:159-164.

Ibrahim, A.H. 2004. Efficacy of exogenous glycine betaine application on sorghum plants grown under salinity stress. Acta Botanica Hungarica 43:307-318.

Kaya, C., Tuna, A.L., and Okant, A.M. 2010. Effect of foliar applied kinetin and indole acetic acid on maize plants grown under saline conditions. Turkish Journal of Agriculture and Forestry 34:529-538.

Kim, Y., Arihara, J., Nakayama, T., Nakayama, N., Shimada, S., and Usui, K. 2004. Antioxidative responses and their relation to salt tolerance in Echinochloa oryzicola Vasing and Setaria virdis (L.) Beauv. Plant Growth Regulation 44:87-92.

Mahajan, S., and Tuteja, N. 2005. Cold, salinity and drought stresses: an overview. Archives of Biochemistry and Biophysics 444:139-158.

Munns, R. 2002. Comparative physiology of salt and water stress. Plant Cell and Environment 25:239-250.

Munns, R., and Tester, M. 2008. Mechanisms of salinity tolerance. Annual Review of Plant Biology 59:651-681.

Murillo, A.B., Lopez, A.R., Kaja, C., Larrinaga, M.J., and Flores-Hernandez, H.A. 2002. Comparative effects of NaCl and polyethylene glycol on germination, emergence and seedling growth of cowpea. Journal of Agronomy and Crop Science 188:235-247

Nimir, N.E.A., Zhou, G., Guo, W., Ma, B., Lu, S., and Wang, Y. 2017. Effect of foliar application of GA, kinetin, and salicylic acid on ions content, membrane permeability, and photosynthesis under salt stress of sweet sorghum [Sorghum bicolor (L.) Moench]. Canadian Journal of Plant Science 97(3):525-535. doi.org/10.1139/cjps-2016-0110.

Nyagah, A.H., and Musyimi, D.M. 2009. Effects of sodium chloride solution stress on germination and growth of passion fruits seedlings. Agricultural and Biological Science Journal 4:49-53.

Parashar, A., and Varma, S.K. 1988. Effect of pre-sowing seed soaking in gibberellic acid, duration of soaking, different temperatures and their interaction on seed germination and early seedling growth of wheat under saline conditions. Plant Physiology and Biochemistry 15:189-197.

Parida, A.K., Das, A.B., and Mohanty, P. 2004. Investigations on the antioxidative defense responses to NaCl stress in amangrove, Bruguiera parviflora: differential regulations of iso-forms of some antioxidative enzymes. Plant Growth Regulation 42:213-226.

Pedranzani, H., Racagni, G., and Alemano, S. 2003. Salt tolerant tomato plants show increased levels of jasmonic acid. Plant Growth Regulation 41:149-158.

Qin, J., Dong, W.Y., He, K.N., Yu, Y., Tan, G.D., Han, L., et al. 2010. NaCl salinity-induced changes in water status, ion contents and photosynthetic properties of Shepherdia argentea (Pursh) Nutt. seedlings. Plant, Soil and Environment 56:325-332.

Ratanavathi, C.V., Dayakar, R.B., and Seet, H.N. 2004. Sweet sorghum: A new raw material for fuel alcohol, in study report on technological aspects in manufacturing ethyl alcohol from cereal grains in Maharashtra. Part II. Department of Scientific \& Industrial Research, Ministry of Science \& Technology, Government of India, New Delhi and Mitcon Consultancy Services Limited, Pune, India.

Romero, A.R., Soria, T., and Cuartero, J. 2001. Tomato plant water uptake and plant water relationships under saline growth conditions. Plant Science 160:265-272.

Saberali, S.F., and Moradi, M. 2017. Effect of salinity on germination and seedling growth of Trigonella foenumgraecum, Dracocephalum moldavica, Satureja hortensis and Anethum graveolens. Journal of the Saudi Society of Agricultural Sciences (In Press).

Srivastava, N.K., and Srivastava, A.K. 2007. Influence of gibberellic acid on ${ }^{14} \mathrm{CO}_{2}$ metabolism, growth and production of alkaloids in Catharanthus roseus. Photosynthetica 45:156-160.

Sudhir, P., and Murthy, S.D.S. 2004. Effects of salt stress on basic processes of photosynthesis. Photosynthetica 42:481-486.

Sun, T.P. 2008. Gibberellin metabolism, perception and signaling pathways in Arabidopsis. The Arabidopsis Book 6:e0103.

Tang, Q.Y., and Feng, M.G. 1997. Practical statistics and DPS data processing system. China Agricultural Press, Beijing, China [in Chinese]. 
Tavakkoli, E., Rengasamy, P., and Mc Donald, G.K. 2010. High concentrations of $\mathrm{Na}^{+}$and $\mathrm{Cl}^{-}$ions in soil solution have simultaneous detrimental effects on growth of faba bean under salinity stress. Journal of Experimental Botany 61:4449-4459.

Tuna, A.L., Cengiz, K., Murat, D., and David, H. 2008. The combined effects of gibberellic acid and salinity on some antioxidant enzyme activities, plant growth parameters and nutritional status in maize plants. Environmental and Experimental Botany 62:1-9.

Willenborg, C.J., Gulden, R.H., Johnson, E.N., and Shirtliffe, S.J. 2004. Germination characteristics of polymer-coated canola (Brassica napus L.) seeds subjected to moisture stress at different temperatures. Agronomy Journal 96:786-791.

Yang, C.J., and Li, G.Y. 2014. Effect of $\mathrm{NaCl}$ stress on germination of birch seeds. Chemical and Pharmaceutical Research 6:1980-1986.

Yildirim, E., Karlidag, H., and Turan, M. 2009. Mitigation of salt stress in strawberry by foliar K, Ca and Mg nutrient supply. Plant, Soil and Environment 55:213-221.

Zhou, G., Nimir, N., Lu, S., Zhai, F., and Wang, Y. 2014. Gibberellic acid and salinity affected growth and antioxidant enzyme activities in castor bean plants at early growth stage. Agronomy Journal 106(4):1340. doi:10.2134/agronj14.0044. 